\title{
The parathyroid is a target organ for FGF23 in rats
}

\author{
Iddo Z. Ben-Dov, ${ }^{1}$ Hillel Galitzer, ${ }^{1}$ Vardit Lavi-Moshayoff, ${ }^{1}$ Regina Goetz, ${ }^{2}$ \\ Makoto Kuro-o,, ${ }^{3}$ Moosa Mohammadi, ${ }^{2}$ Roy Sirkis, ${ }^{4}$ Tally Naveh-Many, ${ }^{1}$ and Justin Silver ${ }^{1}$ \\ 1Minerva Center for Calcium and Bone Metabolism, Nephrology Services, Hadassah Hebrew University Medical Center, Jerusalem, Israel. \\ 2Department of Pharmacology, New York University School of Medicine, New York, New York, USA. ${ }^{3}$ Department of Pathology, \\ The University of Texas Southwestern Medical Center, Dallas, Texas, USA. ${ }^{4}$ ProChon Biotech Ltd., Weizmann Science Park, Ness Ziona, Israel.
}

\begin{abstract}
Phosphate homeostasis is maintained by a counterbalance between efflux from the kidney and influx from intestine and bone. FGF23 is a bone-derived phosphaturic hormone that acts on the kidney to increase phosphate excretion and suppress biosynthesis of vitamin D. FGF23 signals with highest efficacy through several FGF receptors (FGFRs) bound by the transmembrane protein Klotho as a coreceptor. Since most tissues express FGFR, expression of Klotho determines FGF23 target organs. Here we identify the parathyroid as a target organ for FGF23 in rats. We show that the parathyroid gland expressed Klotho and 2 FGFRs. The administration of recombinant FGF23 led to an increase in parathyroid Klotho levels. In addition, FGF23 activated the MAPK pathway in the parathyroid through ERK1/2 phosphorylation and increased early growth response $1 \mathrm{mRNA}$ levels. Using both rats and in vitro rat parathyroid cultures, we show that FGF23 suppressed both parathyroid hormone (PTH) secretion and PTH gene expression. The FGF23-induced decrease in PTH secretion was prevented by a MAPK inhibitor. These data indicate that FGF23 acts directly on the parathyroid through the MAPK pathway to decrease serum PTH. This bone-parathyroid endocrine axis adds a new dimension to the understanding of mineral homeostasis.
\end{abstract}

\section{Introduction}

FGF23 is secreted by osteocytes (1) and osteoblasts (2) in response to high serum phosphate levels and after the administration of the vitamin D metabolite, $1,25(\mathrm{OH})_{2}$-vitamin D (2-4). FGF23 acts on its target tissues by binding to and activating its cognate FGF receptors (FGFRs) in the presence of its obligatory coreceptor, Klotho $(5,6)$. Klotho is a transmembrane protein and acts as a cofactor for FGF23 to facilitate the binding of FGF23 to FGFR1c, $-3 c$, and $-4(5,6)$. The expression of Klotho determines the tissue specificity of its ligand FGF23. Klotho is expressed in the renal distal tubule, the parathyroid, pituitary, and sinoatrial node of the heart (7). In the kidney, FGF23 inhibits phosphate reabsorption and decreases the synthesis of $1,25(\mathrm{OH})_{2}$-vitamin D (8). Membrane-bound Klotho is present in the distal tubule and inhibits the activity of the $\mathrm{Na}-\mathrm{Pi}$ cotransporter in the proximal tubule $(9,10)$, which may be due to the action of a secreted form of Klotho (11). Klotho has weak glucuronidase enzymatic activity and in the renal distal tubule hydrolyzes extracellular sugar residues on the transient receptor potential ion channel $\mathrm{V}$, which leads to increased calcium transport in the kidney (10). In the heart, klotho gene expression was essential for the sinoatrial node to function as a dependable pacemaker under conditions of stress (7). In both the choroid plexus and parathyroid, Klotho protein has been shown to interact with $\mathrm{Na}^{+}, \mathrm{K}^{+}$-ATPase and may be associated with parathyroid hormone (PTH) secretion (12). Klotho also exists as a soluble circulating protein. Administration of FGF23 to rats increased early growth response 1 (Egr-1) mRNA levels in

Nonstandard abbreviations used: ADHR, autosomal dominant hypophosphatemic rickets; $\mathrm{CaR}, \mathrm{Ca}^{2+}$ receptor; Egr-1, early growth response 1; FGFR, FGF receptor; PTH, parathyroid hormone; qRT-PCR, quantitative RT-PCR.

Conflict of interest: The authors have declared that no conflict of interest exists. Citation for this article: J. Clin. Invest. 117:4003-4008 (2007). doi:10.1172/JCI32409. kidney and also in the parathyroid and pituitary (6). We have now studied the function of FGF23 on the parathyroid.

Transgenic mice with FGF23 driven by the $\beta$-actin promoter showed all the biochemical and skeletal phenotypes seen in patients with autosomal dominant hypophosphatemic rickets (ADHR) or tumor-induced osteomalacia, diseases that result from marked increases in mutant or intact FGF23 $(13,14)$. These mice had decreased serum phosphate and $1,25(\mathrm{OH})_{2}$-vitamin D levels, which would be expected to increase PTH secretion. However, PTH levels were decreased (15). Three other studies with genetic manipulation of FGF23 showed increased serum PTH levels (16-18). In 2 of these studies, the increase in serum PTH was attributed to the decrease in serum $1,25(\mathrm{OH})_{2}$-vitamin D $(16,17)$. We show that Klotho is expressed in the parathyroid and that FGF23 activates the MAPK pathway and potently decreases PTH gene expression and secretion both in vivo and in vitro. Hence, we portray an endocrinological axis linking bone cells and the parathyroid, in which the parathyroid is a new target organ for FGF23. FGF23 acts directly on the parathyroid to decrease serum PTH in addition to its actions on the kidney to enhance phosphaturia and inhibit vitamin D metabolism. This novel endocrinological axis is important for the physiologic responses of the parathyroid, kidney, and bone in phosphorus homeostasis.

\section{Results}

We first demonstrated the expression of Klotho in the parathyroid. Immunoblots with anti-Klotho antibody showed Klotho expression in rat microdissected parathyroid. There was no expression of Klotho protein in the thyroid (Figure 1A) or liver (data not shown). Quantitative RT-PCR (qRT-PCR) for Klotho mRNA showed high expression in parathyroid and kidney with negligible expression in thyroid, duodenum, and liver and no expression at all in the spleen (Figure 1B). Immunohistochemistry confirmed the localization of Klotho to the parathyroid and not the surrounding thyroid tissue 
A

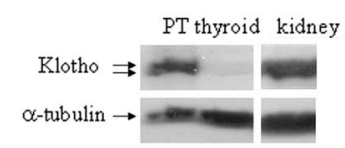

C

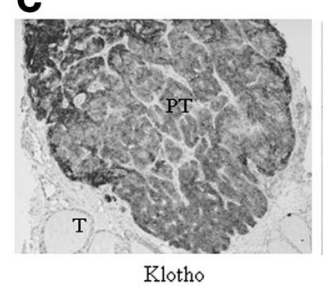

B

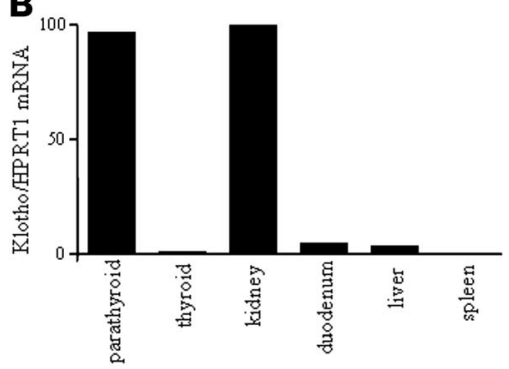

Figure 1

Klotho is expressed in the rat parathyroid. (A) Immunoblot of microdissected parathyroid (PT), thyroid, and kidney using antibodies for Klotho and $\alpha$-tubulin as loading control. (B) Real-time RT-PCR for membrane Klotho mRNA corrected for HPRT1 mRNA from microdissected parathyroid, thyroid, kidney, duodenum, liver, and spleen. Results are expressed as a percentage of expression in total kidney. Klotho mRNA expression is restricted to the parathyroid and kidney. (C) Immunohistochemistry with antibody for Klotho, FGFR1, and FGFR3 in thyroparathyroid tissue, showing specific expression of Klotho protein in the parathyroid and not in the surrounding thyroid (T) tissue. FGFR1 and -3 are also expressed in the parathyroid. A negative control without the primary antibody showed no staining (data not shown). Original magnification, $\times 100$.
(Figure 1C) and also showed the presence of FGFR1 (Figure 1D) and FGFR3 (Figure 1E) in the parathyroid tissue. These results show that the parathyroid expresses the FGFR-Klotho receptor complex and suggest that the parathyroid is a target organ for FGF23.

To study the effect of FGF23 on parathyroid function, rats were injected i.p. with full-length FGF23 harboring ADHR mutations

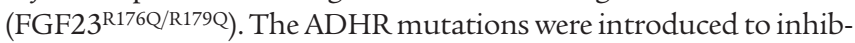
it proteolytic inactivation of FGF23 and to increase its half-life. As a negative control, we injected FGF23 ${ }^{\text {core }}$, an FGF23 variant lacking C-terminal residues past the ${ }^{176} \mathrm{RXXR}{ }^{179}$ cleavage site for furin-like proteases (19). This C-terminal truncation inactivates FGF23 by abrogating binding to Klotho (19). FGF23 ${ }^{\mathrm{R} 176 \mathrm{Q} / \mathrm{R} 179 \mathrm{Q}}$ or FGF23 ${ }^{\text {core }}$ were injected by daily i.p. injections for 5 days. Serum biochemistry showed the expected decrease in $1,25(\mathrm{OH})_{2}$-vitamin D levels in

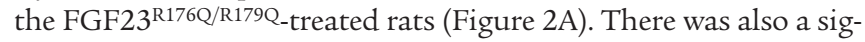
nificant decrease in serum phosphorus (control, $3.03 \pm 0.11 \mathrm{mmol} / 1$ $(n=9)$; FGF23 treated, $2.64 \pm 0.02 \mathrm{mmol} / \mathrm{l}(n=10), P<0.01)$, with no significant changes in serum calcium (data not shown). Importantly, serum PTH was decreased after FGF23 ${ }^{\mathrm{R} 176 \mathrm{Q} / \mathrm{R} 179 \mathrm{Q}}$ administration when compared with FGF23 ${ }^{\text {core }}$ or HEPES (Figure 2A). FGF23 $2176 \mathrm{Q} / \mathrm{R} 179 \mathrm{Q}$ also led to a marked decrease in PTH mRNA levels as measured by northern blot and quantified by qRT-PCR (Figure 2B). Immunoblots

\section{Figure 2}

FGF23 ${ }^{\mathrm{R} 176 \mathrm{Q} / \mathrm{R} 179 \mathrm{Q}}$ decreases serum 1,25(OH $)_{2}-$ vitamin D, serum PTH, and PTH mRNA levels; increases parathyroid Klotho protein; and induces ERK phosphorylation in long-term experiments. FGF23R176Q/R179Q (FGF23), FGF23core, or HEPES (control) were given daily for 5 days. (A) Serum 1,25(OH $)_{2}$-vitamin D and PTH levels were decreased by FGF23R176Q/R179Q compared with FGF23core or HEPES. The results are expressed as mean \pm SEM $(n=5)$. ${ }^{*} P<0.05$ compared with control or FGF23 ${ }^{\text {core }}$. (B) FGF23R176Q/R179Q decreased PTH mRNA levels as measured by northern blot (a pool from 3 rats in each lane) and by qRT-PCR. The results are expressed as mean $\pm \operatorname{SEM}(n=5)$. ${ }^{*} P<0.05$. (C) Immunoblots for Klotho, phospho-ERK1/2, total ERK $1 / 2$, and $\alpha$-tubulin of microdissected parathyroids from rats given $\mathrm{FGF} 23^{\mathrm{R} 176 \mathrm{Q} / \mathrm{R} 179 \mathrm{Q}}$ or FGF23 ${ }^{\text {core }}$ as described above. Each lane represents parathyroid extracts from a single rat. Results from 2 rats are shown for FGF23 ${ }^{\text {core }}$ and 3 rats for FGF23 R176Q/R179Q. All samples were run on the same blot.
A
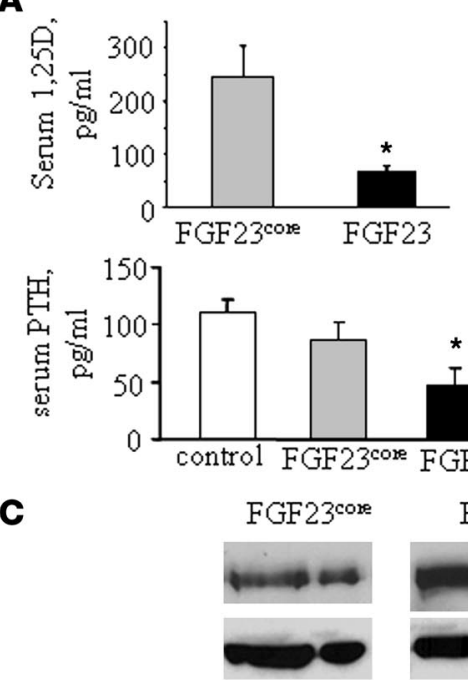

Klotho/a-tubulin: $1 \pm 0.1$

(fold increase)

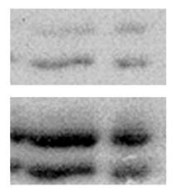

$1 \pm 0.1$

pERK $1 / 2$ / ERK $1 / 2$

(fold increase)
B
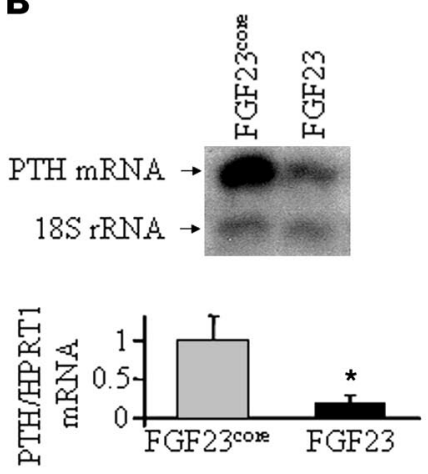

\section{FGF23}

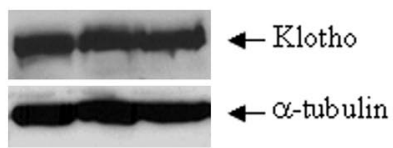

$1.3 \pm 0.1$ *

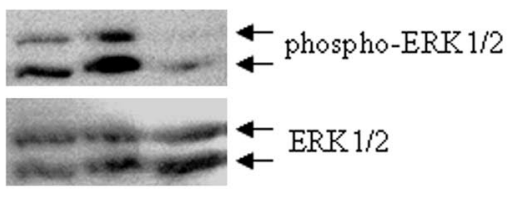

$3.1 \pm 0.8^{*}$ 
A

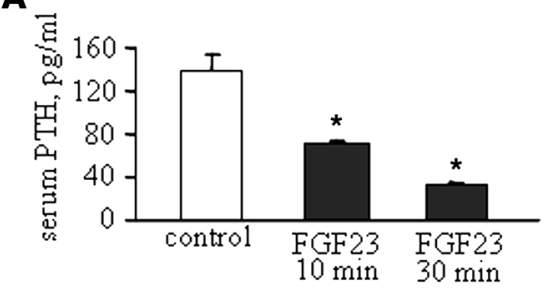

C

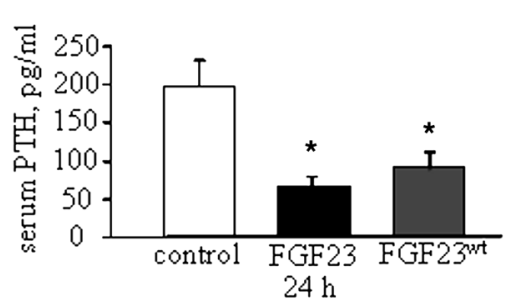

B

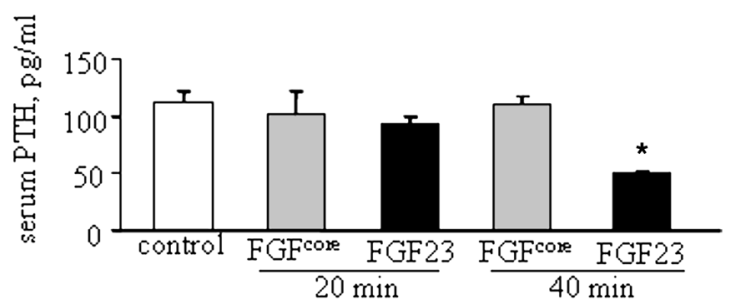

D

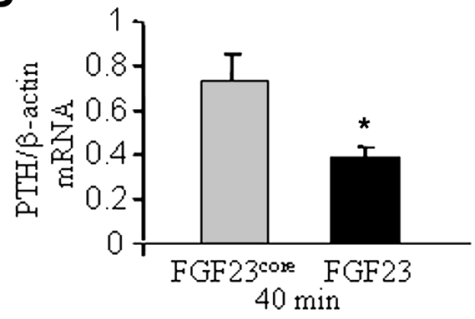

\section{Figure 3}

FGF23 decreases serum PTH and PTH mRNA levels in vivo in short-term experiments. FGF23R176Q/R179Q, FGF23core, FGF23 wild-type (FGF23wt), or HEPES were injected i.v. or i.p. into 4 rats per group. At the indicated times, serum PTH levels were measured and parathyroids extracted for RNA analysis. (A) Serum PTH levels at 10 and 30 minutes after i.v. FGF23R176Q/R179Q or HEPES administration. (B) Serum PTH levels from untreated rats (control) and rats 20 or 40 minutes after i.p. FGF23R176Q/R179Q or FGF23core treatment. (C) Serum PTH at 24 hours after i.p. HEPES, FGF23 ${ }^{\text {176Q/R179Q }}$, or FGF23 ${ }^{\text {wt }}$ administration. (D) qRT-PCR analysis for PTH mRNA of RNA extracted from microdissected parathyroids 40 minutes after FGF23 $176 \mathrm{Q} / \mathrm{R} 179 \mathrm{Q}$ or FGF23 core treatment. FGF23R176Q/R179Q and FGF23wt administration decreased serum PTH and PTH mRNA levels. ${ }^{\star} P<0.05$ compared with FGF23core and (where applicable) controls.

of parathyroid glands from rats given FGF23 $3^{\mathrm{R} 176 \mathrm{Q} / \mathrm{R} 179 \mathrm{Q}}$ for 5 days showed an increase in Klotho protein (Figure 2C). FGF23 acts on the Klotho-FGFR through the MAPK pathway in cells transfected with Klotho as well as in the kidney, as shown by an increased phosphorylation of ERK1/2 $(5,20)$. FGF23 $3^{\mathrm{R} 176 \mathrm{Q} / \mathrm{R} 179 \mathrm{Q}}$ led to an increase in phospho-ERK1/2 in the parathyroid gland (Figure 2C), indicating activation of the MAPK pathway in the FGF23-treated rats.

We then performed short-term experiments to better define the FGF23 signal transduction in the parathyroid and its effect on PTH secretion. FGF23 $3^{\mathrm{R} 176 \mathrm{Q} / \mathrm{R} 179 \mathrm{Q}}$ decreased serum PTH at 10 and 30 minutes when given i.v. (Figure 3A). FGF23 ${ }^{\mathrm{R} 176 \mathrm{Q} / \mathrm{R} 179 \mathrm{Q}}$ given i.p. led to a decrease in serum PTH at 40 minutes when compared with FGF23 ${ }^{\text {core }}$ or untreated rats (Figure 3B). Similarly, FGF23 ${ }^{\text {R176Q/R179Q }}$ given i.p. decreased serum PTH at 24 hours (Figure 3C). Wildtype FGF23 without the R176Q/R179Q mutation also decreased

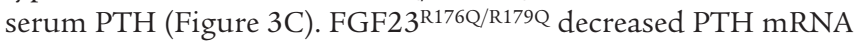
levels at 40 minutes, as shown by qRT-PCR (Figure 3D). Therefore, FGF23 decreased PTH secretion and mRNA levels in shortterm experiments. To show that the MAPK pathway is required for the FGF23 effect on PTH secretion, we used the MAPK inhibitor U0126. We devised what we believe to be a novel method where FGF23 was given i.v., and the inhibitor U0126 was added topically. Rat parathyroid glands were exposed and submerged in PBS with or without the ERK1/2 inhibitor (U0126), immediately followed by i.v. injection of FGF23 or HEPES carrier. Serum PTH levels were increased by U0126 at 5 and 20 minutes. FGF23 decreased serum PTH levels at 20 minutes in the absence of the inhibitor (Figure 4), as before (Figure 3A). Importantly, U0126 prevented the FGF23induced decrease in serum PTH (Figure 4). However, PTH levels after FGF23 administration in the presence of the inhibitor U0126 did not reach the level induced by U0126 alone. These results indicate that the MAPK pathway in the parathyroid is important in maintaining a tonic suppression of PTH secretion. This may represent basal activity of the $\mathrm{Ca}^{2+}$ receptor (CaR), FGF23 action, and other factors that decrease serum PTH through the MAPK pathway. To separate the direct effects of the MAPK inhibitor from other humoral factors, we then performed in vitro studies.

In the absence of a parathyroid cell line, we studied the direct action of FGF23 on in vitro organ cultures of rat parathyroids. Microdissected parathyroid glands were incubated in a medium with FGF23 ${ }^{\mathrm{R} 176 \mathrm{Q} / \mathrm{R} 179 \mathrm{Q}}$ or FGF23 ${ }^{\text {core }}$. Egr-1 mRNA levels at 10 minutes were increased in the parathyroids incubated with FGF23 R176Q/R179Q (Figure 5A). FGF23 ${ }^{\mathrm{R} 176 \mathrm{Q} / \mathrm{R} 179 \mathrm{Q}}$ significantly decreased PTH secretion at 20,40, and 60 minutes compared with FGF23 ${ }^{\text {core }}$ control(Figure 5B).

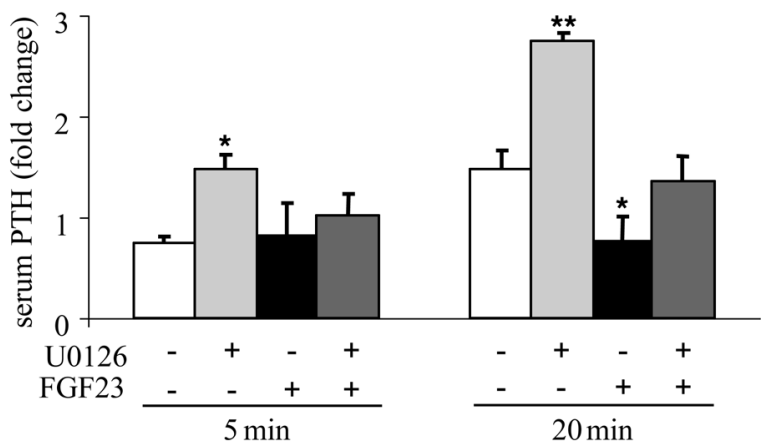

\section{Figure 4}

Local exposure to the MAPK inhibitor U0126 prevents i.v. FGF23R176Q/R179Q from decreasing serum PTH levels. Rat parathyroid glands were exposed and submerged in PBS with or without ERK1/2 inhibitor (U0126, $1 \mu \mathrm{M})$, immediately followed by i.v. injection of FGF23R176Q/R179Q (+; $5 \mathrm{ng} / \mathrm{rat})$ or HEPES carrier (-). Serum PTH levels at 5 and 20 minutes were normalized to pretreatment baseline values $(n=4$ in each group). ${ }^{\star} P<0.05 ;{ }^{\star \star} P<0.005$ compared to the control group at each time point. 
A

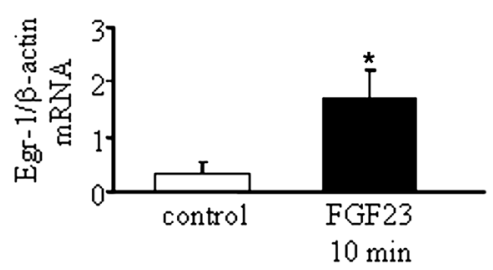

B

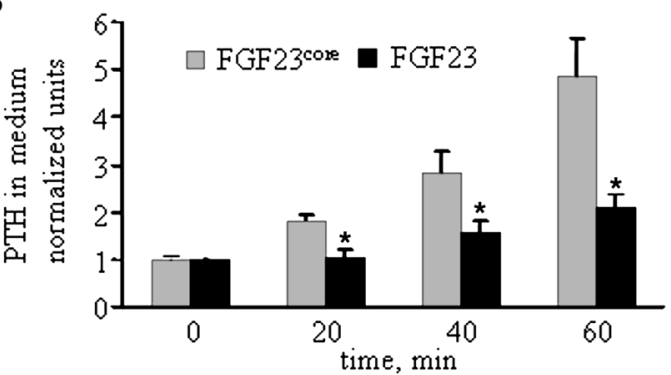

C

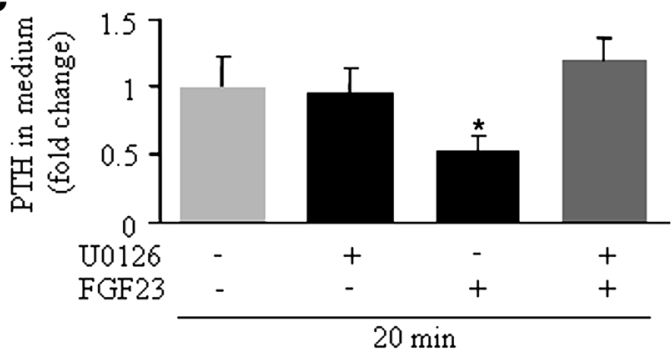

We next studied the effect of the MAPK inhibitor U0126 on PTH secretion in response to FGF23 (Figure 5C). U0126 alone had no effect on PTH secretion, in contrast the increased PTH secretion it caused when applied topically in vivo. FGF23 decreased PTH secretion as shown in Figure 5. Importantly, U0126 prevented FGF23 from causing decreased PTH secretion (Figure 5C). Therefore, FGF23 acted directly on the parathyroid to activate the MAPK pathway and decrease PTH secretion both in vivo (Figure 4) and in vitro (Figure 5).

\section{Discussion}

The FGF23-Klotho pathway is crucial to life. Mice with genetic deletion of Klotho age and die prematurely and have diffuse vascular calcification due to increased levels of serum phosphate and $1,25(\mathrm{OH})_{2}$-vitamin D (5). Their serum FGF23 levels are markedly increased. Feeding these Klotho ${ }^{-/}$mice with a diet deficient in vitamin D rescues most of the phenotype (21). A recent study using FGF23-null mice showed that a vitamin D-deficient diet improved survival but did not prevent vascular calcification (22). In contrast, when these FGF23-null mice were fed a phosphorus-depleted diet, both survival and vascular calcification were improved, implying that phosphorus retention was the major factor responsible for vascular calcification (22).

The receptor for FGF23 is the heterodimer of the coreceptor Klotho, with the FGFR types $1 c, 3 c$, and 4 (5). We now show that the parathyroid is also a target organ for FGF23. We confirm that Klotho is expressed in the parathyroid (7) and show that FGFR1 and -3 are also expressed in the parathyroid. We show that the expression of Klotho is increased by FGF23. The upregulation of Klotho may be a function of feedback regulation of the FGFR by its ligand, FGF23.

\section{Figure 5}

FGF23 decreases PTH secretion in vitro in isolated rat parathyroid glands. (A) Egr-1 mRNA levels. Pairs of parathyroid glands were preincubated for 1 hour and then incubated without or with FGF23R176Q/R179Q for 10 minutes. Glands were extracted and analyzed by qRT-PCR for Egr-1 and $\beta$-actin. ${ }^{*} P<0.05$. (B) Parathyroid glands from each rat were preincubated in medium for 60 minutes (time 0 ) and then transferred to medium containing either FGF23 ${ }^{\text {core }}$ or FGF23 ${ }^{\text {R176Q/R179Q }}$ ( $n=13$ rats per group). Media were sampled for PTH at the indicated time points. The accumulated PTH level of each parathyroid gland pair was measured and normalized to PTH in the medium of the glands at time 0. PTH secretion was inhibited by FGF23R176Q/R179Q compared with FGF23 ${ }^{\text {core }}$ at 20,40 , and 60 minutes. ${ }^{*} P<0.05$. (C) As described above, pairs of parathyroid glands were incubated in control medium or in medium with U0126, FGF23 ${ }^{\text {176Q/R179Q }}$ alone, or FGF23 ${ }^{\text {R176Q/R179Q }}$ with U0126 ( $n=7,6,7$, and 4 rats, respectively). Medium was sampled for PTH at 20 minutes and is presented as fold change in PTH levels compared with time 0 . ${ }^{*} P<0.05$ for the comparison with control and with the U0126 + FGF23 treatment group.

FGF23 decreased PTH mRNA and serum PTH in both shortterm and long-term experiments. In short-term experiments, the catalytically stable form of FGF23 and the wild-type FGF23 decreased serum PTH levels, establishing the physiological relevance of the action of FGF23 in decreasing PTH gene expression. Urakawa et al. (6) showed that i.v. FGF23 administration increases parathyroid Egr-1 mRNA levels at 30 minutes, implying that the MAPK pathway is either directly or indirectly involved in the effect that FGF23 has on the parathyroid. We showed that FGF23 acts directly on the parathyroid by using an in vitro organ culture system of microdissected parathyroid glands in medium, using FGF23 or FGF23 ${ }^{\text {core }}$ as control. This organ culture system was used because there is no parathyroid cell line. FGF23 led to an increase in Egr-1 mRNA at 10 minutes and a decrease in secreted PTH that accumulated in the medium at 20,40, and 60 minutes. This shows that FGF23 acts directly on the parathyroid to activate the MAPK pathway and decrease PTH secretion.

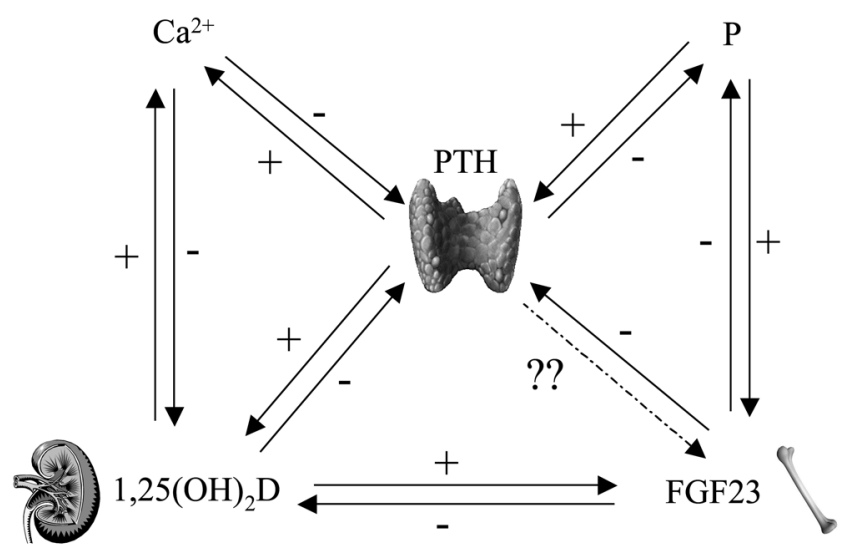

\section{Figure 6}

Endocrinological feedback loops in mineral homeostasis. There are endocrinologic feedback loops between $\mathrm{PTH}, \mathrm{Ca}^{2+}$, phosphorus, and $1,25(\mathrm{OH})_{2}$-vitamin D. Phosphorus and $1,25(\mathrm{OH})_{2}$-vitamin D levels are both trophic to FGF23, which in turn decreases phosphorus and $1,25(\mathrm{OH})_{2}$-vitamin D levels. We now show that FGF23 decreases serum PTH and PTH gene expression by activating its cognate FGFRs in the parathyroid in a Klotho-dependent fashion. 
Table 1

Primers used for qRT-PCR

\begin{tabular}{lcc} 
Primer & Forward & Reverse \\
PTH & TTGTCTCCTTACCCAGGCAGAT & TTTGCCCAGGTTGTGCATAA \\
HPRT1 & CCCAGCGTCGTGATTAGTGA & CCAAATCTTCAGCATAATGATTAGGTAT \\
$\beta$-Actin & CAGGCATTGCTGACAGGATG & CTCAGGAGGAGCAATGATCTTGAT \\
Egr-1 & TACGAGCACCTGACCACAGAGT & GCTGGGATAACTTGTCTCCACC \\
\hline
\end{tabular}

(28). This paradox was explained in part by the finding that calreticulin prevents the binding of the VDR to the VDRE of the PTH promoter (34). The resistance of the parathyroid to elevated levels of FGF23 in uremia remains to be studied. In summary, we propose what we believe to be a new endocrinological bone-parathyroid axis in which FGF23 acts on the parathyroid. As components of endocrinological feedback loops ensuring physiological $\mathrm{Ca}^{2+}$ and

We studied whether the MAPK system plays a role in the parathyroid in vivo and in vitro by using an ERK1/2 inhibitor. To do this we utilized what is, to our knowledge, a novel in vivo experimental method in which the thyroparathyroid glands were exposed in anesthetized rats. The ERK1/2 inhibitor U0126 was applied topically on the thyroparathyroids in vivo. This method maintained the glands in their physiological milieus. FGF23 was then given i.v. and serum PTH levels measured. Interestingly, the ERK1/2 inhibitor alone increased serum PTH levels, implying that MAPK tonically restrains PTH secretion. In part, this may reflect activity of the CaR in the parathyroid (23) as well as other autocrine and humoral factors that decrease PTH secretion, such endothelin and acidic FGF $(24,25)$. U0126 prevented the decrease in serum PTH that had resulted from injection of FGF23 but did not restore PTH to the levels found when U0126 was given alone. This suggests that in vivo inhibition of the MAPK pathway partially prevents the decrease in serum PTH that results from FGF23. In vitro, interestingly, U0126 did not increase secreted PTH as it did in vivo. This may be because the isolated parathyroid is not exposed to the other humoral factors that are known to act on the parathyroid to decrease PTH secretion through the MAPK pathway. Therefore, the isolated parathyroid glands in vitro provide a better system to study the role of the MAPK pathway in FGF23 signaling in the parathyroid. Indeed, the effect of FGF23 was abolished by the restoration by U0126 of secreted PTH to basal levels. Together with the FGF23-induced increase in Egr-1 mRNA levels and ERK phosphorylation, these results indicate that FGF23 acts through the MAPK pathway to decrease PTH secretion.

In the parathyroid, a high-serum $\mathrm{Ca}^{2+}$ leads to activation of the $\mathrm{CaR}$ on the parathyroid cell membrane which activates the MAPK pathway as well as PLC $\gamma$ with the subsequent mobilization of $\mathrm{Ca}^{2+}$ from the endoplasmic reticulum $(23,26)$. The net result is a decrease in PTH gene expression, secretion and PT cell proliferation (27-29). It is tempting to speculate that the FGF23-KlothoFGFR and the $\mathrm{Ca}^{2+}-\mathrm{CaR}$ systems may act in concert through the MAPK pathway to decrease PTH gene expression and secretion. The parathyroid $\mathrm{CaR}$ responds to changes in serum $\mathrm{Ca}^{2+}$ at the levels of PTH gene expression, secretion and PT cell proliferation. In the secondary hyperparathyroidism of chronic renal failure there is down-regulation of the parathyroid $\mathrm{CaR}(30)$ and vitamin $\mathrm{D}$ receptor (31). 1,25(OH) ${ }_{2}$-vitamin D decreases PTH gene transcription together with upregulation of the vitamin D receptor $(32,33)$.

The finding that FGF23 decreases PTH gene expression and secretion is surprising when one considers that in renal failure there is a consistent increase in both FGF23 and serum PTH. A similar paradox in parathyroid pathophysiology occurs in chronic hypocalcemia, in which there are markedly elevated levels of serum calcitriol, which would be expected to decrease serum PTH levels. Despite the high-serum calcitriol, levels of serum PTH are increased phosphate homeostasis, FGF23, 1,25(OH $)_{2}$-vitamin D, and $\mathrm{Ca}^{2+}$ all act to decrease PTH synthesis and secretion (Figure 6).

\section{Methods}

Animal experiments. FGF23 $3^{\mathrm{R} 176 \mathrm{Q} / \mathrm{R} 179 \mathrm{Q}}$ and FGF23 $3^{\text {core }}$ (19) and wild-type FGF23 recombinant proteins were prepared in E. coli. The FGF23 ${ }^{\mathrm{R} 176 \mathrm{Q} / \mathrm{R} 179 \mathrm{Q}}$ mutation is present in patients with excessive activity of FGF23 because of an aberration in the proteolytic cleavage site (13). The FGF23 core lacks the carboxyterminal sequence and is biologically inactive. The FGF23 compounds were injected i.p. or i.v. into Sabra rats (200 g) fed a control diet as a single dose $(15 \mu \mathrm{g} / \mathrm{rat})$ or i.p. daily $(7.5 \mu \mathrm{g} / \mathrm{rat} / \mathrm{d})$ for 5 days. For all experiments, 4-5 rats were used in each group. Each experiment was repeated at least 3 times. Serum biochemistry for $\mathrm{Ca}^{2+}$, phosphorus, creatinine, and $1,25(\mathrm{OH})_{2}$-vitamin D were measured as previously described (35). FGF23 (Kainos Lab) (36) and PTH (Immutopics) were measured in sera. Parathyroid glands were microdissected and homogenized. RNA was extracted for northern blots and real-time RT-PCR, and proteins were extracted for western blots, as previously described (37). In some experiments thyroid, liver, kidney, duodenum, and spleen were also analyzed. All animal experiments were approved by the Hadassah Hebrew University Animal Care and Use Committee.

In vitro organ culture experiments. Intact microdissected parathyroid glands were studied using a modification of the method of Rodriguez et al. (38). In brief, intact microdissected parathyroid glands were preincubated for 20 minutes in individual wells on an 8- $\mu \mathrm{m}$ Nucleopore track-etch filter (Whatman catalog no. 110414) floating on $2 \mathrm{ml}$ of incubation medium (1.0 $\mathrm{mM} \mathrm{CaCl}_{2}$, $1 \mathrm{mM}$ Na-pyruvate, $4 \mathrm{mM}$ glutamine, $0.1 \mathrm{IU} / \mathrm{ml}$ human insulin, $0.1 \%$ bovine serum albumin) in DMEM pH 7.4, at $37^{\circ} \mathrm{C}$. The glands were then transferred with the filter to medium containing FGF23 $3^{\mathrm{R} 176 \mathrm{Q} / \mathrm{R} 179 \mathrm{Q}}$ or FGF23 $3^{\text {core }}(100 \mathrm{ng} / \mathrm{ml})$ or control medium for the times indicated in Figures 2-4, at which time medium was sampled for PTH analysis and RNA extracted at the end of the incubations.

In vivo FGF23 and local exposure to U0126. The parathyroid glands of anesthetized rats were exposed and submerged in PBS with or without ERK1/2 inhibitor (U0126, $1 \mu \mathrm{M}$ ), immediately followed by i.v. injection of FGF23 ( $5 \mathrm{ng} / \mathrm{rat}$ ) or HEPES carrier. Blood samples were taken at 5 and 20 minutes for PTH analysis. In some experiments, U0126 $(1 \mu \mathrm{M})$ was added to the medium with or without FGF23.

Protein and RNA analysis and immunobistochemistry. Microdissected parathyroid tissue from individual rats was homogenized and extracted in TRI reagent (Molecular Research Center), yielding both RNA and proteins from the same sample. Proteins were analyzed by western blots using antibodies for Klotho (Kyowa Hakko Kogyo), phospho-ERK, ERK1/2, and $\alpha$-tubulin (Sigma-Aldrich). RNA was analyzed by northern blots for PTH mRNA and 18S rRNA and by qRT-PCR for PTH, Egr-1 mRNA, and control mRNA. Real-time PCR was conducted using ABI Prism 7900 Sequence Detection System (Applied Biosystems) and SYBR Green Mix (Applied Biosystems). Primers for rat PTH and the control genes $\beta$-actin and HPRT1, which we found to be similarly expressed across the study groups, spanned large 
intronic areas to prevent amplification of genomic DNA. Amplicon specificity was ascertained by melting curve analysis as well as length evaluation by ethidium bromide staining of agarose gels. The primers used for PCR are shown in Table 1. The primers were designed using Primer Express software version 2.0 (Applied Biosystems). Quantification was done by the standard-curve method.

Immunohistochemistry was performed on paraffin-embedded parathyroid tissue. Following microdissection, parathyroid glands from normal rats were placed in $4 \%$ formaldehyde. Paraffin tissue sections (4-6 $\mu \mathrm{m})$ were deparaffinized and incubated overnight at $4{ }^{\circ} \mathrm{C}$ with rat anti-Klotho antibody (Kyowa Hakko Kogyo), rabbit anti-FgfR1 antibody, or rabbit anti-FgfR3 antibody (Santa Cruz Biotechnology Inc.). $\mathrm{DAB}$ reagent was applied after incubation with the appropriate HRPbound secondary antibody.

Statistics. Values are reported as mean \pm SEM unless stated otherwise. One-way ANOVA was used to assess differences from the control group. For the ex vivo analysis of Egr-1 mRNA, the Mann-Whitney $U$ test was used (SPSS 13.0; SPSS Inc.). A 2-tailed $P$ value was considered significant when less than 0.05 .

1. Liu, S., et al. 2006. Pathogenic role of Fgf23 in Hyp mice. Am. J. Physiol. Endocrinol. Metab. 291:E38-E49.

2. Masuyama, R., et al. 2006. Vitamin D receptor in chondrocytes promotes osteoclastogenesis and regulates FGF23 production in osteoblasts. J. Clin. Invest. 116:3150-3159. doi:10.1172/JCI29463.

3. Kolek, O.I., et al. 2005. 1alpha,25-Dihydroxyvitamin D3 upregulates FGF23 gene expression in bone: the final link in a renal-gastrointestinal-skeletal axis that controls phosphate transport. Am. J. Physiol. Gastrointest. Liver Physiol. 289:G1036-G1042.

4. Liu, S., et al. 2006. Fibroblast growth factor 23 is a counter-regulatory phosphaturic hormone for vitamin D. J. Am. Soc. Nephrol. 17:1305-1315.

5. Kurosu, H., et al. 2006. Regulation of fibroblast growth factor-23 signaling by klotho. J. Biol. Chem. 281:6120-6123.

6. Urakawa, I., et al. 2006. Klotho converts canonical FGF receptor into a specific receptor for FGF23. Nature. 444:770-774.

7. Takeshita, K., et al. 2004. Sinoatrial node dysfunction and early unexpected death of mice with a defect of klotho gene expression. Circulation. 109:1776-1782.

8. Saito, H., et al. 2003. Human fibroblast growth factor-23 mutants suppress $\mathrm{Na}+$-dependent phosphate co-transport activity and 1alpha,25dihydroxyvitamin D3 production. J. Biol. Chem. 278:2206-2211.

9. Li, S.A., et al. 2004. Immunohistochemical localization of Klotho protein in brain, kidney, and reproductive organs of mice. Cell Struct. Funct. 29:91-99.

10. Chang, Q., et al. 2005. The beta-glucuronidase klotho hydrolyzes and activates the TRPV5 channel. Science. 310:490-493.

11. Imura, A., et al. 2004. Secreted Klotho protein in sera and CSF: implication for post-translational cleavage in release of Klotho protein from cell membrane. FEBS Lett. 565:143-147.

12. Imura, A., et al. 2007. alpha-Klotho as a regulator of calcium homeostasis. Science. 316:1615-1618.

13. White, K.E., et al. 2000. Autosomal dominant hypophosphataemic rickets is associated with mutations in FGF23. Nat. Genet. 26:345-348.

14. White, K.E., et al. 2001. The autosomal dominant hypophosphatemic rickets (ADHR) gene is a secreted polypeptide overexpressed by tumors that cause phosphate wasting. J. Clin. Endocrinol. Metab. 86:497-500.

15. Shimada, T., et al. 2004. FGF-23 transgenic mice demonstrate hypophosphatemic rickets with reduced expression of sodium phosphate cotrans-

\section{Acknowledgments}

This work was supported in part by grants from the Israel Academy of Sciences (to T. Naveh-Many), the Minerva Center for Calcium and Bone Metabolism (to T. Naveh-Many and J. Silver), the NIH (DE13686; to M. Mohammadi), and the Irma T. Hirschl Fund (to M. Mohammadi). The Minerva Center for Calcium and Bone Metabolism is funded through the German Federal Ministry of Education and Research (BMBF).

Received for publication April 16, 2007, and accepted in revised form September 5, 2007.

Address correspondence to: Justin Silver, Minerva Center for Calcium and Bone Metabolism, Nephrology Services, Hadassah Hospital, PO Box 12000, Jerusalem 91120, Israel. Phone: 972-2-6436778; Fax: 972-2-6421234; E-mail: silver@huji.ac.il.

Iddo Z. Ben-Dov and Hillel Galitzer contributed equally to this work.

porter type IIa. Biochem. Biophys. Res. Commun. 314:409-414

16. Bai, X.Y., Miao, D., Goltzman, D., and Karaplis, A.C. 2003. The autosomal dominant hypophosphatemic rickets R176Q mutation in fibroblast growth factor 23 resists proteolytic cleavage and enhances in vivo biological potency. J. Biol.Chem. 278:9843-9849.

17. Bai, X., Miao, D., Li, J., Goltzman, D., and Karaplis, A.C. 2004. Transgenic mice overexpressing human fibroblast growth factor 23 (R176Q) delineate a putative role for parathyroid hormone in renal phosphate wasting disorders. Endocrinology. 145:5269-5279.

18. Larsson, T., et al. 2004. Transgenic mice expressing fibroblast growth factor 23 under the control of the alpha1(I) collagen promoter exhibit growth retardation, osteomalacia, and disturbed phosphate homeostasis. Endocrinology. 145:3087-3094.

19. Goetz, R., et al. 2007. Molecular insights into the klotho-dependent, endocrine mode of action of FGF19 subfamily members. Mol. Cell. Biol. 27:3417-3428.

20. Yamashita, T., Konishi, M., Miyake, A., Inui, K., and Itoh, N. 2002. Fibroblast growth factor (FGF)-23 inhibits renal phosphate reabsorption by activation of the mitogen-activated protein kinase pathway. J. Biol. Chem. 277:28265-28270.

21. Tsujikawa, H., Kurotaki, Y., Fujimori, T., Fukuda, K., and Nabeshima, Y. 2003. Klotho, a gene related to a syndrome resembling human premature aging, functions in a negative regulatory circuit of vitamin D endocrine system. Mol. Endocrinol. 17:2393-2403.

22. Stubbs, J.R., et al. 2007. Role of hyperphosphatemia and 1,25-dihydroxyvitamin $\mathrm{D}$ in vascular calcification and mortality in fibroblastic growth factor 23 null mice. J. Am. Soc. Nephrol. 18:2116-2124.

23. Hofer, A.M., and Brown, E.M. 2003. Extracellular calcium sensing and signalling. Nat. Rev. Mol. Cell Biol. 4:530-538.

24. Fujii, Y., et al. 1991. Endothelin as an autocrine factor in the regulation of parathyroid cells. Proc. Natl. Acad. Sci. U. S. A. 88:4235-4239.

25. Sakaguchi, K. 1992. Acidic fibroblast growth factor autocrine system as a mediator of calciumregulated parathyroid cell growth. J. Biol. Chem. 267:24554-24562.

26. Brown, E.M., et al. 1993. Cloning and characterization of an extracellular $\mathrm{Ca}^{2+}$-sensing receptor from bovine parathyroid. Nature. 366:575-580.

27. Silver, J., Kilav, R., and Naveh-Many, T. 2002. Mech- anisms of secondary hyperparathyroidism. Am. J. Physiol. Renal Physiol. 283:F367-F376.

28. Naveh-Many, T., and Silver, J. 1990. Regulation of parathyroid hormone gene expression by hypocalcemia, hypercalcemia, and vitamin D in the rat. J. Clin. Invest. 86:1313-1319.

29. Naveh-Many, T., Rahamimov, R., Livni, N., and Silver, J. 1995. Parathyroid cell proliferation in normal and chronic renal failure rats: the effects of calcium, phosphate and vitamin D. J. Clin. Invest. 96:1786-1793.

30. Carling, T., Rastad, J., Szabo, E., Westin, G., and Akerstrom, G. 2000. Reduced parathyroid vitamin $D$ receptor messenger ribonucleic acid levels in primary and secondary hyperparathyroidism. J. Clin. Endocrinol. Metab. 85:2000-2003.

31. Fukuda, N., et al. 1993. Decreased 1,25-dihydroxyvitamin D3 receptor density is associated with a more severe form of parathyroid hyperplasia in chronic uremic patients. J. Clin. Invest. 92:1436-1443.

32. Silver, J., Naveh-Many, T., Mayer, H., Schmelzer, H.J., and Popovtzer, M.M. 1986. Regulation by vitamin D metabolites of parathyroid hormone gene transcription in vivo in the rat. J. Clin. Invest. 78:1296-1301.

33. Naveh-Many, T., Marx, R., Keshet, E., Pike, J.W., and Silver, J. 1990. Regulation of 1,25-dihydroxyvitamin D3 receptor gene expression by 1,25-dihydroxyvitamin D3 in the parathyroid in vivo. J. Clin. Invest. 86:1968-1975.

34. Sela-Brown, A., et al. 1998. Calreticulin inhibits vitamin D's action on the PTH gene in vitro and may prevent vitamin D's effect in vivo in hypocalcemic rats. Mol. Endocrinol. 12:1193-1200.

35. Ben Dov, I.Z., et al. 2007. Lanthanum carbonate decreases PTH gene expression with no hepatotoxicity in uraemic rats. Nephrol. Dial.Transplant. 22:362-368.

36. Kato, Y., et al. 2000. Establishment of the antiKlotho monoclonal antibodies and detection of Klotho protein in kidneys. Biochem. Biophys. Res. Commun. 267:597-602.

37. Levi, R., et al. 2006. Increased parathyroid hormone gene expression in secondary hyperparathyroidism of experimental uremia is reversed by calcimimetics: correlation with posttranslational modification of the trans acting factor AUF1. J. Am. Soc. Nephrol. 17:107-112.

38. Rodriguez, M.E., et al. 2007. The calcimimetic $\mathrm{R}-568$ increases vitamin $\mathrm{D}$ receptor expression in rat parathyroid glands. Am. J. Physiol. Renal Physiol. 292:F1390-F1395. 